\title{
Fisioterapia en Salud Mental: Antecedentes históricos.
}

\author{
Daniel Jesús Catalán Matamoros ${ }^{1}$ \\ https://orcid.org/0000-0002-3086-6812 \\ Universidad Carlos III de Madrid \\ Universidad de Almería, España
}

Fecha de recepción: 01 de abril de 2019

\begin{abstract}
Fecha de aprobación: 01 de junio de 2019
Resumen

La Fisioterapia en Salud Mental (FSM) es una especialidad de la fisioterapia que abarca un amplio espectro de técnicas dirigidas directamente a mejorar los trastornos mentales. En países europeos tales como Suecia, Noruega, Bélgica e Inglaterra es habitual encontrar fisioterapeutas integrados en los equipos de salud mental. Este artículo muestra los antecedentes históricos de la FSM para ofrecer al fisioterapeuta una base teórico-conceptual y constituir así un punto de partida para la puesta en marcha de nuevas experiencias prácticas y/o ensayos clínicos en este campo. Desde la Edad Antigua se tiene evidencia del uso de diversos agentes físicos para el tratamiento de trastornos mentales, pero no es hasta el s. XX cuando se desarrollan los antecedentes históricos directos de la FSM. Éstos provienen de diversas áreas de conocimiento tales como la psicoterapia corporal, corrientes filosóficas y artes expresivas. Desde mediados del s. XX, los fisioterapeutas han adaptado estos conocimientos al propio campo de actuación para crear así la especialidad de Fisioterapia en Salud Mental.
\end{abstract}

Palabras clave: fisioterapia; salud mental; psiquiatría; historia.

\section{Physiotherapy in Mental Health: Historical background}

\begin{abstract}
Physiotherapy in Mental Health (PMH) is a physiotherapy specialty that encompasses a high number of techniques directly applied to improve mental disorders. In European countries like Sweden, Norway, Belgium and England is a common fact to find physiotherapists working within mental health teams. This article shows the historical background of MHP to give to the physiotherapist a theoretical framework and to offer a starting point in order to create new experiences and/or clinical trials in this field. From the Ancient World there is evidence of the use of physical agents to treat mental disorders, but the most important historical background was originated in the XXth century. This comes from different fields of knowledge like body psychotherapy, philosophical traditions and expressive arts. Since 1950, physiotherapists have adapted this knowledge to the own practice to create the specialty in Physiotherapy in Mental Health.
\end{abstract}

Keywords: physical therapy (specialty), mental health, psychiatry, history.

\footnotetext{
${ }^{1}$ Correo de contacto: dcatalan@ual.es

DOI: 10.30788/RevColReh.v18.n2.2019.394
}

Esta obra se encuentra bajo licencia internacional CC BY 4.0 Institución editora: Escuela Colombiana de Rehabilitación 


\section{Introducción}

La Fisioterapia ofrece un enfoque holístico a la prevención, diagnóstico y tratamiento de las alteraciones del movimiento y mejora la capacidad funcional para aumentar la salud y el bienestar de la comunidad desde una perspectiva tanto individual como poblacional. Es una parte integral del sistema sanitario actual (Fary, 2003). La fisioterapia es una ciencia que puede ser aplicada en una amplia gama de áreas o especialidades sanitarias. De manera tradicional los fisioterapeutas han intervenido mayoritariamente en las áreas de traumatología, neurología y reumatología, pero existen otras en donde la Fisioterapia puede también ofrecer efectividad y en ciertos países se puede observar la figura del fisioterapeuta como miembro del equipo multidisciplinar encargado de dar respuesta a nuevos retos que se van planteando en otras áreas de salud, como, por ejemplo, oncología, nefrología, estética y salud mental.

La FSM es una especialidad de la fisioterapia que abarca un amplio espectro de técnicas dirigidas directamente a mejorar los trastornos mentales. En países europeos tales como Suecia, Noruega, Bélgica e Inglaterra es habitual encontrar fisioterapeutas integrados en los equipos de salud mental. Esta especialidad se basa fundamentalmente en el concepto biopsicosocial de salud y más concretamente al concepto de intervención terapéutica denominado "cuerpo-mente". Cada vez más estudios muestran que el bienestar físico es un pre-requisito para el bienestar mental y viceversa. De hecho, estudios serios recientes han demostrado una clara correlación entre ejercicio físico y la reducción de ansiedad y depresión especialmente en población con alteraciones mentales. Donaghy y Durward (2000) definen la figura del fisioterapeuta en salud mental como: "el profesional que puede ofrecer un gran arsenal de abordajes físicos de tratamiento dirigidos a relajar síntomas y mejorar la calidad de vida. Por lo tanto, el fisioterapeuta aporta un gran apoyo en la evaluación y tratamiento de pacientes mentales que es normalmente ofrecido en conjunción con el tratamiento farmacológico prescrito y el psicoterapéutico en el contexto del equipo interdisciplinar"

Dentro del grupo de las enfermedades mentales y psicosomáticas, existen experiencias con intervenciones fisioterapéuticas en las siguientes enfermedades: (Donaghy et al, 2000; Dragesund y Raheim; 2006; Probst, 1997; Friis, Skatteboe, Kvamsdal y Vaglum, 1989; Everett, Donaghy y Feaver, 2003; Degener, 2006; Hulting, 2006) a) Esquizofrenia; b) Demencias; c) Depresión; d) Ansiedad; e) Dependencia y abuso de substancias; f) alteraciones de la personalidad; g) Trastornos de la alimentación; h) Síndrome de fatiga crónica; i) Autolesiones y j) Fibromialgia.

Este artículo presenta los antecedentes históricos de la FSM, los cuales, debido a ser una especialidad relativamente reciente en Fisioterapia, tiene sus antecedentes más directos en otras áreas del conocimiento tales como la psicoterapia corporal, las corrientes filosóficas y las artes expresivas.

El objetivo fundamental de este artículo es presentar los antecedentes históricos de la Fisioterapia en Salud Mental para poder crear así una base teórica y científica de dicha especialidad de la Fisioterapia.

\section{Método}

Se han realizado búsquedas de información en diversas bases de datos nacionales e internacionales (AMED, EMBASE, CINAHL, MEDLINE, PsycINFO, ISI-Web of Knowledge - Web of Science, IME, IBECS y PEDro). Se ha consultado el libro de resúmenes de las Conferencias Internacionales en 
Fisioterapia en Psiquiatría y Salud Mental celebradas por la IOPTMH, y también se han realizado entrevistas en profundidad con fisioterapeutas especialistas en Salud Mental, concretamente con Liv Helvik Skjaerven, Marit Nilsen y Ulla-Britt Skatteboe.

Los criterios de inclusión de información estuvieron abiertos a todos aquellos formatos con información relacionada a la Fisioterapia en Salud Mental y sus antecedentes históricos.

\section{Resultados}

\section{Edad Antigua}

Las civilizaciones antiguas tenían una concepción mágico-animista o demonológica de gran parte de las enfermedades, en especial de los trastornos psíquicos. Es así como en Mesopotamia los primeros médicos babilónicos fueron los sacerdotes de Assipu, que se ocupaban de las enfermedades internas y, especialmente, de las afecciones mentales, que eran consideradas como posesiones demoníacas y tratadas con métodos mágico-religiosos. Por otra parte, no se distinguía entre enfermedad psíquica y enfermedad somática y, en su actuación terapéutica el "brujo-curador-terapeuta" utilizaba medios variados mágicos, ritos, interrogatorios, adivinatorios, transferencias y exorcismos; psicológicos como la catarsis y la sugestión inherente a toda relación y ni que decir tiene de otros medios de naturaleza física masajes y agentes térmicos y química como son sustancias vegetales, minerales y animales que se encontraban a su alcance, desarrollando una aproximación holística a la enfermedad y al tratamiento (Yusta, 1996)

Desde tiempo inmemorial la humanidad ha utilizado los agentes físicos para el alivio de sus enfermedades. Los primeros escritos al respecto aparecen en China (2500 a.C.) en torno al Kung-Fu. Por su parte, los hindúes hablan del yoga y de los masajes como técnica de relajación y purificación (1800 a.C). En la India antigua hacia el 1800 a. de J.C., el Ayurveda, IV volumen del Atharva-Veda, muestra cómo se contemplaban en la persona diferentes órdenes de realidad física, psicológica, social y metafísica. El objetivo era el conocimiento total de la persona y no exclusivamente su enfermedad, es decir, se realizaba un abordaje de naturaleza biopsicosocial, incluso, más amplio, geográfico y cultural. Entre otros medios terapéuticos, el ejercicio y el masaje eran recomendados en los procesos reumáticos crónicos, de interés actual para la psicosomática. Posteriormente en Grecia aparecen los Ascetas que desarrollan el concepto "cuerpo y mente", siendo los filósofos los que se dedicaban al tratamiento de los problemas mentales.

Hipócrates (460-380 a.C.) cambió el concepto de la práctica médica. Profundizó en el uso de los agentes físicos (masaje, agua, ejercicio, etc.) en diversas patologías. En relación con las alteraciones mentales Hipócrates recomendaba el agua fría como sedante para la melancolía. La termoterapia se usaba tanto en Grecia como en Roma como medio para aliviar tensiones tanto físicas como mentales. 


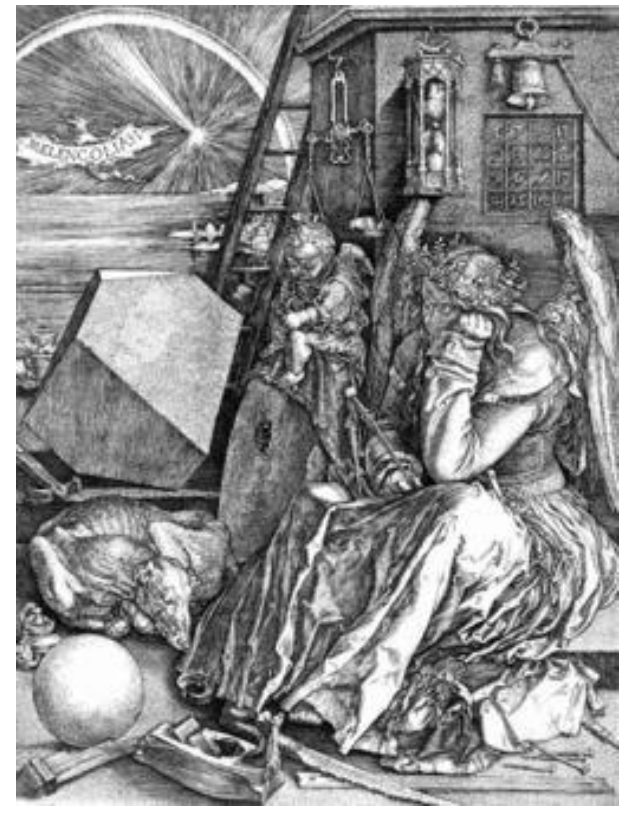

Figura 1. Melancholia de Albrecht Dürer
Los romanos siguieron directrices similares a las griegas, utilizando el ejercicio físico como forma de bienestar general, entendiendo éste también desde el punto de vista mental. Entre sus máximos exponentes en ésta área se encuentra Celso (25 a.C. - 50 d.C.), conocido como el "Hipócrates latino", quien dividió las enfermedades en locales y generales, incluyendo entre estas últimas a las enfermedades mentales, que a su vez las dividió en febriles (delirios) y no febriles (locura). Recomendaba la hidroterapia para la locura triste. Areteo (50-130 d.C.), que hizo descripciones clínicas de diversas enfermedades y se preocupó por el bienestar de los pacientes, encontró que la manía y la melancolía podían presentarse como parte de una misma enfermedad, y usaba el agua fría para el frenesí y el síncope.

\section{Edad Media}

Tras la caída del Imperio Romano, durante la Edad Media, las prometedoras ideas de las culturas griega y latina sufren una involución. La iglesia excluyó a la psiquiatría de la medicina, pero no pudo abolirla, pues reapareció bajo el nombre de demonología dando paso a la aparición de la brujería. Esta situación persiste durante el Renacimiento, Barroco y la Ilustración, siendo en esta última época cuando, aunque los enfermos mentales ya no eran quemados en la hoguera, su suerte era aún lamentable ya que si no eran internados en los hospitales, vagaban solitarios, siendo objeto de desprecios, burlas y maltratos.

Pero no todo fue entonces negativo para la salud mental, ya que en este periodo acontecen también algunos hechos importantes, como la Primera Revolución Psiquiátrica, iniciada por el sacerdote Fray Juan Gilbert Jofré que, en 1409, crea en Valencia el primer hospital psiquiátrico del mundo. Paracelso (1493-1541) se opuso duramente a las creencias médicas de su época. Rechazó la demonología, así también lo hizo Vives (1492-1540), considerado como el padre de la psiquiatría moderna y primer psiquiatra.

De esta manera, las terapias físicas también sufren una involución debido al catolicismo y su gran limitación impuesta consistente en la prohibición de mostrar el cuerpo y poder recibir tratamientos o placeres en él, por ser considerado causa suficiente de pecado.

\section{Siglo XVI - Siglo XIX}

En la Época Romántica, alrededor de 1800 comienza en Francia la Psiquiatría Científica, con la obra del médico y reformador francés Philippe Pinel (1745-1826). La contribución fundamental de Pinel fue cambiar la actitud de la sociedad hacia los enfermos mentales para que fueran considerados como seres humanos merecedores de un tratamiento sanitario. Observó que sus pacientes psiquiátricos mejoraban 
con actividades como la pintura y otras formas expresivas. Se empieza a observar cómo los pacientes con trastornos mentales necesitan de varios tipos de terapias expresivas. Con la obra de Pinel y sus seguidores, como Esquirol, la psiquiatría se libera de las interpretaciones demonológicas y se sustituye la especulación por la observación empírica, originándose así la Segunda Revolución Psiquiátrica.

Es aquí cuando de nuevo, los agentes físicos y los aspectos físicos de la Psiquiatría comienzan a retomar la importancia necesaria para su estudio y uso por parte de los investigadores de la época. La electricidad, el masaje, el ejercicio físico, el calor, el frío, etc. empiezan a estudiarse desde un punto de vista empírico.

El cirujano inglés James Braid (1795-1860) descubrió que los estados de trance, como los inducidos por Mesmer, no son por magia ni magnetismo, sino por exceso de fatiga muscular debida a prolongados periodos de concentración, acuñando el término "hipnosis". Este procedimiento lo utilizó en cirugía para disminuir el dolor. El neurólogo francés Jean Martin Charcot (1825-1893) fue el primero en estudiar la función del trauma psicológico en el origen de la histeria, suponiendo que los recuerdos traumáticos se almacenan en el inconsciente, separados de la conciencia, y dan lugar a los síntomas físicos.

Paralelamente el estudio de los agentes físicos va profundizándose en Europa, así Per Henrik Ling (1776-1839) crea los postulados del "masaje clásico", Duchene de Boulonge (1806-1875) usa las corrientes galvánicas como curación y realiza el diagnóstico mediante corrientes eléctricas.

\section{Siglos XX y XXI}

Sin lugar a dudas, el Siglo XX ha sido clave tanto para la fisioterapia como para la salud mental donde han alcanzado el máximo desarrollo conseguido hasta ahora. Por lo tanto, es también donde más ha avanzado el estudio y la aplicación de la FSM.

Desde mediados del siglo XIX, diferentes tipos de terapias centradas en el cuerpo han sido desarrolladas y usadas tanto en ámbitos sanitarios como psicoterapéuticos. Conciencia corporal y funcionamiento motor de diferentes grupos de pacientes han sido estudiados con más o menos profundidad y descritos por muchos autores en bibliografía psiquiátrica (Roxendal, 1985). Shilder (1950) analizó la imagen que los pacientes tienen de su propio cuerpo, así como los bordes del cuerpo percibidos en aquellos que sufrían de esquizofrenia, daño cerebral, amputaciones, comparándolos con sujetos sanos. Fisher y Cleveland, (1958), encontraron que los pacientes con esquizofrenia percibían los bordes de su cuerpo de una forma indefinida al ser comparados con sujetos sanos.

En el último siglo se han desarrollado diversas terapias centradas en el cuerpo y modalidades en el campo sanitario, psicoterapéutico y de desarrollo personal en el mundo occidental (Lundvik, 2001). Estudios clínicos indican que muchas de ellas son efectivas, cada una de una forma diferente. Todas tienen un punto en común, el movimiento. De hecho en FSM el movimiento humano es una parte esencial tanto en el diagnóstico como en la intervención fisioterapéutica.

Mathias Alexander fue un actor que desarrolló la técnica de tratamiento "Alexander" en Australia en los comienzos de 1900. Desarrolló un sistema de ejercicios considerando el patrón del movimiento como un todo, tomando como punto de inicio la posición de la cabeza y del cuello (Alexander, 1932). De acuerdo con la técnica Alexander, en los tratamientos de problemas mentales nunca se puede olvidar el patrón corporal usado, "solamente después de mejorar patrones posturales que han sido trabajados, 
queda claro como los trastornos mentales son influidos por patrones posturales inadecuados" (Bott, 2006)

Moshe Feldenkrais desarrolló un sistema de movimientos para todas las regiones corporales llamado "Método Feldenkrais" (Lundvik, 2001). Sus fundamentos teóricos se basan en asociaciones de situaciones emocionales o experiencias desagradables con ciertos comportamientos. Según Feldenkrais, la forma del movimiento se adquiere mediante repetición y que el aprendizaje del patrón corporal sea realizado de forma conjunta al tratamiento psiquiátrico para que los efectos perduren más tiempo y para completar la madurez del individuo (Lundvik, 2001).

La FSM ha tomado conocimientos de otras disciplinas pudiéndose englobar en tres: corrientes filosóficas, psicoterapia corporal y artes expresivas.

Corrientes filosóficas: Rudolf Steiner (1861-1925) fue un científico, filósofo y creador de un sistema llamado antroposofía. Creó estilos de vida alternativos en diversas áreas como en la agricultura biodinámica, la medicina antroposófica, y un sistema de movimientos llamado Euritmia. La Euritmia se centra sobre todo en el equilibrio, postura, movimientos corporales y en la voz. En el ámbito de la medicina de orientación antroposófica (Steiner, 1974) se han desarrollado múltiples terapias complementarias reconocidas (también a nivel oficial, sobre todo en Alemania, Austria y Suiza) como la euritmia curativa, la terapia artística (modelado y pintura), la musicoterapia, la terapia de la palabra y el masaje rítmico. La presencia/conciencia en los movimientos fueron considerados esenciales para aumentar la calidad del movimiento (Steiner, 1974).

Por su parte, el filósofo alemán Edmund Husserl (1859-1938) comenzó el desarrollo de la fenomenología la cual ha tenido mucha importancia en el desarrollo de la investigación cualitativa. Es un método filosófico que procede a partir del análisis intuitivo de los objetos tal como son dados a la conciencia cognoscente, a partir de lo cual busca inferir los rasgos esenciales de la experiencia y lo experimentado. Husserl analizó la estructura de los actos mentales y cómo se dirigen a objetos reales e irreales, destacando el concepto de intencionalidad que infiere siempre a la conciencia, dirigiéndose hacia algo que ha sido experimentado con especial significado para el individuo.

Vinculado a la fenomenología, el filósofo francés Maurice Merleau-Ponty (1908-1961), también llamado "el filósofo del cuerpo", expuso la ambigüedad de la naturaleza humana. El cuerpo puede tener dos sensaciones, la de sujeto y la de objeto. Esta doble interpretación también se refiere a la vida, el mundo de la vida, ya que también está lleno de experiencias objetivas y subjetivas. Nosotros estamos en el mundo como un cuerpo vivo que se abre hacía el mundo y le da significado (Merleau-Ponty, 1962)

Yuasa Yasuo (1987, citado por Skjaerven, 1999) es un filósofo japonés contemporáneo con un conocimiento profundo sobre la filosofía fenomenológica/existencialista. Su filosofía tiene orígenes en la meditación Zen. La filosofía asiática considera la unidad mente-cuerpo como algo que se debe mantener mediante la práctica. Yuasa mantiene que la sabiduría no se puede adquirir únicamente mediante un conocimiento intelectual, ya que necesita del cuerpo para adquirirse. Cultivar el cuerpo consiste en adquirir nuevas experiencias (Skjaerven, 1999)

El psicoanálisis corporal: Freud (1923) se pregunta sobre la relación existente entre el cuerpo y la personalidad y concluye que: «Él Yo es sobre todo una esencia-cuerpo, no es sólo una esenciasuperficie, sino, él mismo, la proyección de una superficie». Esta farragosa expresión viene a destacar la cualidad corporal del Yo desde los inicios de la vida psíquica, el Yo es ante todo un Yo corporal. Los 
primeros estudios de Freud del impacto corporal sobre la psique influyeron a sus discípulos como a Reich que desarrolló un enfoque más físico de la psicoterapia (Mattsson \& Mattsson, 1994; Reich, 1949) Bülow-Hansen y Heir-Bunkan (fisioterapeutas), Waahl (psiquiatra pediátrico) y Braatöy (neurólogo/psiquiatra) han desarrollado y perfeccionado el trabajo de Reich en sus respectivos campos (Reich, 1949)

En relación a la psicoterapia centrada en el cuerpo, Wilhelm Reich (1897-1957) introdujo el trabajo con el cuerpo en el proceso terapéutico. Fue excluido de la comunidad del psicoanálisis por sus ideas sobre la importancia del cuerpo y sexualidad para la psicopatología. Desarrolló una teoría llamada "Análisis Caracterológico" (Reich, 1970). De esta manera, antes de pretender llegar al centro del problema psíquico del paciente mediante charlas y circunloquios que se prolongaban años y años, la misión de Reich consistía en derribar las barreras que bloqueaban el libre flujo de energía psíquica del paciente. Esto lo consiguió aplicando otro principio descubierto por él que afirmaba: La psique de una persona y su musculatura voluntaria son funcionalmente equivalentes. Esto quiere decir que los bloqueos psíquicos se corresponden a contracciones musculares crónicas. Tratando de explicarlo, pongamos un ejemplo: una persona con miedos tiene perpetuamente tensos los muslos y los hombros, pues es la manera que tiene el cuerpo de prepararse para protegerse la cabeza y echar a correr, reacción lógica ante un peligro. Así,
Reich se dispuso a combatir las enfermedades mentales a través de la liberación de las tensiones musculares crónicas, lo que proporcionó resultados notables (Reich, 1970). Esto molestaba mucho a los psicoanalistas freudianos, que se abstenían completamente del contacto físico con sus pacientes, aun teniendo un contacto emocional (llamado transferencia) por el cual el psicoanalista se convertía en padre simbólico del paciente.

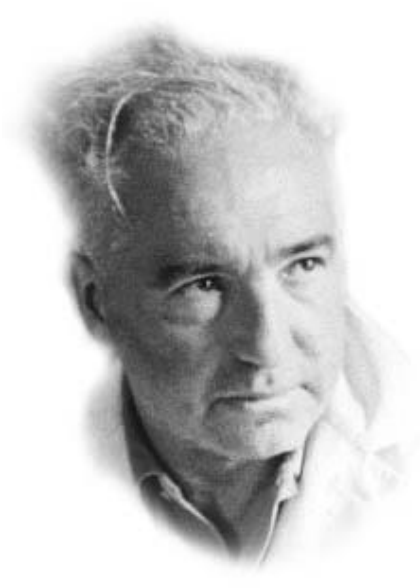

Figura 2. Wilhelm Reich (1897-1957)

En una revisión realizada (Lowe, Tritt, Lahmann \& Röhricht, 2006) sobre estudios sobre métodos de psicoterapia corporal presentaron varias intervenciones realizadas en diversos tipos de patologías. Por ejemplo varios estudios observaron la mejoría en pacientes esquizofrénicos mediante la técnica del ego corporal, la terapia del drama y el movimiento, y también mediante la aplicación de terapia grupal psicoterapéutica centrada en el cuerpo (Goertzel, May, Salkin y Schoop; 1965; Nitsun, Stapleton, Bender; 1974; Maurer-Groeli; 1976)

Alexander Lowen, un discípulo de Reich integró el análisis caraterológico en el campo clínico y desarrolló la teoría de la "Bioenergética" (Lundvik, 2001) que es una conjunción del "análisis caracterológico" planteado por W. Reich y la práctica de ejercicios físicos de tensión y relajación en diversas zonas del cuerpo. Acompañado por propuestas expresivas de diversa índole, Lowen afirmaba que "desde que las funciones de carga y descarga muscular son una unidad, la bioenergética trabaja con las dos para aumentar el nivel de energía de la persona, para mejorar su autoexpresión y mejorar el flujo de sentido en su cuerpo. Por lo tanto, la empatía está siempre en la respiración, sensación y movimiento" (Lowen, 1975). En bioenergética, la falta de energía es considerada como un resultado de tensión muscular crónica causada por la supresión de las emociones. La vida emocional de una persona, su 
espontaneidad y viveza, dependen de la movilidad de su cuerpo, sus movimientos y acciones, de acuerdo a las mismas teorías. La actitud de una persona hacía sí misma se refleja en la postura y en la forma de cómo se mueve (Lowen, 1988). Una de las ideas básicas de Lowen es que el organismo vivo se expresa más claramente a través del movimiento que a través de las palabras (Lowen, 1995).

La pregunta que Lowen se hace es: Si los problemas de la personalidad y del carácter están tan férreamente estructuradas en los movimientos físicos, en la forma de la respiración, en las tensiones musculares, ¿no debería entonces la empresa terapéutica orientarse también al trabajo físico? Gudat, Convent, Heinemann (1997) junto con Ventling y Gerhard (2000) han estudiado la efectividad de la bioenergética en diferentes trastornos observando efectividad especialmente en ansiedad neurótica, depresión y problemas

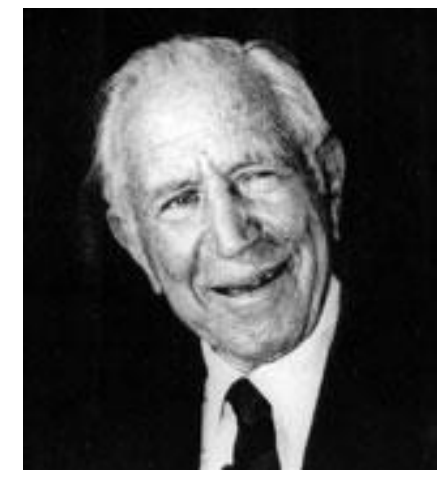

Figura 3. Alexander Lowen psicosomáticos.

Otra técnica que puede ser encuadrada dentro de la psicoterapia corporal es la "relajación funcional" (Lowe et al, 2006) que fue desarrollada entre 1946 y 1956 por Marianne Fuchs y consiste en pequeños movimientos de diversas articulaciones unidos a la respiración. Al mismo tiempo el paciente presta atención al propio cuerpo, en particular en cambios de sensaciones corporales y el proceso corporal autónomo. Estas sensaciones corporales, los cambios y sus significados subjetivos son verbalizados por el paciente mediante el diálogo. Se han realizado diversos estudios observándose efectividad mediante la aplicación de la relajación funcional en patologías tales como asma y otros problemas respiratorios (Deter y Allert, 1983; Loew, Siegfried, Martus, Tritt, Hahn; 1996; Loew, Tritt, Siegfried, Bohmann, Martus, Hahn; 2001) colon irritable (Loew y Rapin, 1994) dolor de cabeza crónico (Loew, Sohn, Martus, Tritt, y Rechlin, 2000)

Otra técnica definida por Lowe et al (2006) como parte de la psicoterapia corporal es la "terapia de la concentración en el movimiento" la cual fue desarrollada por M. Goldberg, G. Heller y H. Stolze. El efecto terapéutico es conseguido por el hecho que el paciente puede mejorar su autoestima mediante la realización de un movimiento con sensibilidad y motivación. Baumann (1994) muestra la efectividad de esta terapia en pacientes con problemas psicosomáticos.

Las artes expresivas: Existen diversas modalidades de artes expresivas basadas en el uso del movimiento y la expresión lo que mejora la interrelación cuerpo-mente; entre ellas se encuentran la musicoterapia, la terapia de la danza, de la pintura, etc.

Dentro del arte de la danza, dos bailarines al comienzo del siglo XX fueron especialmente famosos por su influencia expresiva en la danza. La primera fue Isadora Duncan (1878-1927), quien en su trabajo expresó la necesidad de un significado profundo del interior, y una calidad profunda de la expresión (Duncan, 1938)

Otra bailarina que destacó en estas líneas fue Marta Graham (1894-1991) quien trabajó en América donde desarrolló la danza moderna (Lundvik, 2001). Se interesó en redescubrir la capacidad del 
movimiento del cuerpo, trabajando con los recursos corporales y la elasticidad de los músculos, así como la integración entre respiración y movimientos (Lundvik, 2001).

Martha Graham entendió que cualquier movimiento del cuerpo debía partir de lo que se denomina plexo solar -músculos del abdomen-, centro de energía del ser humano. La lógica de su famosa contracción del cuerpo con el ombligo empujando hacia la espalda, haciéndola cóncava con la presión de los músculos abdominales, práctica que en 1923 Joseph H. Pilates (1880-1967), sin ser bailarín ni coreógrafo, llevó hasta Nueva York con la apertura allí de su estudio para el arte del control del cuerpo. Formó a bailarines jóvenes para su compañía, desarrollando una técnica (la más consistente y completa de la danza moderna estadounidense) que comprendía la contracción y la relajación de distintas partes del cuerpo, una estrecha relación entre respiración y movimiento, unas líneas de cuerpo austeras y angulares, y un contacto muy cercano con el suelo (Baril, 1987).

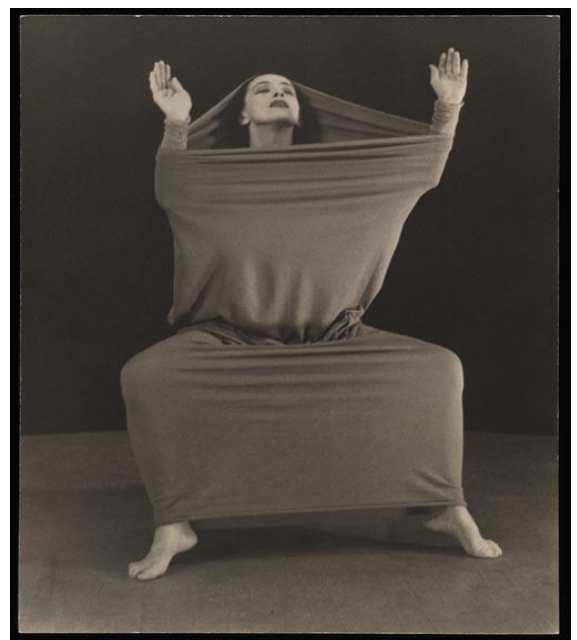

Figura 4. Marta Graham (1894 - 1991)

"Lamentación"

El alemán, Rudolf Laban, bailarín y coreógrafo, trabajó como investigador del movimiento en Inglaterra a mediados del siglo XX. Laban (1960) afirmaba que las formas y los ritmos de los movimientos mostraban las actitudes de la persona, así como también revelaba el humor o rasgos de la personalidad. Laban reconoce ciertas características del movimiento, no únicamente las físicas, sino las emocionales, cognitivas y espiritual. Todas ellas juntas forman un entendimiento completo del hombre (Freud, 1923)

La terapia de la danza intenta iniciar un proceso curativo mediante el movimiento (Lowe et al, 2006). Se utilizan movimientos básicos elementos de la danza mediante la improvisación para conseguir la integración del cuerpo y la mente, mientras que el terapeuta se concentra en el comportamiento, la expresión, la comunicación y la adaptación del paciente. Los conflictos y emociones ocultas salen a la luz y se muestran mediante la integración del movimiento.

Diversos estudios han estudiado la efectividad de la terapia de la danza. En 1988, Lausberg et al aplicaron la terapia de la danza en pacientes con problemas psicosomáticos encontrando cambios en un mejor equilibrio, desarrollo del movimiento, patrón postural, más espacio usado y una reducción distancia hacia el terapeuta. En un estudio realizado en pacientes con trastornos de la alimentación (Larisch, 2006) encontraron que el $81 \%$ de los pacientes estimaron que la terapia de la danza fue importante en su proceso terapéutico.

En 1962, Stanislavski desarrolló teorías del movimiento aplicadas al teatro. Observó cómo los principiantes debían desarrollar un entrenamiento tanto mental como corporal. Desarrolló un entrenamiento para integrar el cuerpo y la mente para encontrar un equilibrio natural, cómodo y presente sobre el escenario. Trabajaba el uso de dibujos para transformar los movimientos y la voz del actor en algo orgánico, natural y libre sobre el escenario. 
La fisioterapia en salud mental. Partiendo de las áreas de conocimiento descritas anteriormente, diversos fisioterapeutas comenzaron a adaptar dichos conocimientos en el campo de la fisioterapia. A lo largo de las últimas décadas, los fisioterapeutas especialistas en salud mental han realizado grandes esfuerzos para profundizar sobre esta disciplina la cual estaba muy poco documentada en sus inicios. Ha sido de gran importancia que fisioterapeutas que han pertenecido al mundo académico y de la investigación hayan mostrado un gran interés en basar en la evidencia científica las intervenciones fisioterapéuticas que se comenzaron a realizar en el ámbito de la salud mental. De esta forma, esta disciplina ha estado tomando mayor importancia en los servicios de salud mental mediante la creación y validación de escalas de evaluación, la determinación de la efectividad de diversas estrategias fisioterapéuticas de intervención en el curso de ciertas alteraciones mentales, y desarrollando programas de formación de pre y posgrado para fisioterapeutas.

Desde los últimos 50 años, ha habido un desarrollo de la fisioterapia dentro del área de la salud mental en los países Escandinavos (Skjaerven, 2006) En Suecia, en la década de 1950, las intervenciones fisioterapéuticas más usadas dentro de los centros psiquiátricos fueron sobre todo técnicas de relajación, como relajación progresiva (Roxendal, 1985), entrenamiento autogénico y diversas intervenciones de actividad física. Inicialmente, el objetivo de la actividad física era fundamentalmente el de mantener el cuerpo en un estado saludable y las habilidades físicas hasta que la salud mental sea restaurada. Actualmente, la actividad física en salud mental ha demostrado mejorar algunos de los síntomas de ciertos trastornos mentales, como por ejemplo estados depresivos.

La FSM plantea que el cuerpo influye tanto en establecer como en mantener síntomas mentales. El impacto en el cuerpo se puede observar por ejemplo en la marcha y en la atención y percepción del paciente al desarrollo de los movimientos. Desde esta perspectiva, la fisioterapia psiquiátrica estimula el paciente hacia experiencias sobre la manifestación de sentimientos a través del cuerpo y sobre el por qué conflictos de larga duración pueden quedarse impresos en el cuerpo. (Mattsson, Egberg, Armelius y Mattsson, 1995)

En línea con estas ideas, se crearon diversas estrategias de intervención fisioterapéutica dirigidas a los trastornos mentales. La fisioterapia psicomotora, creada por la fisioterapeuta noruega Ädel Bülow Hansen y el psiquiatra Braatoy durante el período entre 1946 y 1953 (Skjaerven, 2006; Dragesund \& Raheim, 2006), fue desarrollada en Noruega y es aplicada actualmente en casi todas las unidades de fisioterapia psiquiátrica (Mattsson et al, 1995). Se centra en diferentes niveles de experiencias corporales. Se basa en el planteamiento de que el estrés físico o emocional es expresado en la respiración, musculatura y en los movimientos. A través del trabajo de la postura del paciente, tensión muscular y patrón respiratorio, el fisioterapeuta estimula al cuerpo para estirar, e instaurar movimientos de la respiración correctos a través de masajes en varias regiones corporales y movimientos globales. El objetivo es abrirse a un cambio básico en la postura y una función respiratoria más flexible. Consta de dos instrumentos de evaluación validados: "The Comprehensive Body Examination" (el examen corporal comprensivo) y "The Global Physiotherapy Examination" (el examen de fisioterapia global) (Skjaerven, 2006)

Los fisioterapeutas nórdicos en salud mental utilizan la terapia de la conciencia corporal y la fisioterapia psicomotora siendo ésta última también bastante utilizada por fisioterapeutas belgas. Estas técnicas incluyen el trabajo del equilibrio, movimiento, concentración, masaje, respiración, relajación y conciencia corporal. 
Los fisioterapeutas noruegos Heir-Bunkan (1979) y Johnsen (1975) desarrollaron diversas investigaciones en relación a la terapia psicomotora (Bunkan, 1979; Johnsen, 1995) Según la teoría de la fisioterapia psicomotora, cuando un sujeto se mantiene en bipedestación, el patrón de su respiración presenta aspectos característicos de la relación cuerpo-mente (Mattsson et al, 1995). Los sujetos con buena situación psico-social y psicológica también tienen una buena función corporal global (Mattsson et al, 1994; Mattsson et al, 1995).

En Suecia, la fisioterapeuta Gertrud Roxendal desarrolló otra estrategia fisioterapéutica en psiquiatría, la Terapia de la Conciencia Corporal (TCC) que es uno de los métodos que actualmente más se aplica en fisioterapia psiquiátrica (Roxendal G. (1985). Estas técnicas representan una visión holística del proceso terapéutico y están basadas en los siguientes aspectos fundamentales (Mattsson et al, 1995):

- $\quad$ El cuerpo forma una unidad funcional y co-operante.

- Cuerpo y mente (psyche y soma) interactúan recíprocamente.

A principios de 1970, en Inglaterra, los fisioterapeutas que trabajaban en salud mental enfocaron su trabajo en aumentar la movilidad y la función de los mayores con demencia y problemas mentales crónicos (Donaghy et al, 2000). Desde estos años, las funciones de los fisioterapeutas ingleses en salud mental han ido aumentando incluyendo evaluaciones y tratamientos de pacientes con trastornos psicosomáticos, ansiedad, depresión, esquizofrenia, daño cerebral adquirido, alteraciones de la alimentación y adicciones (Everett, Dennis y Rickets, 1995).

En Fisioterapia Psiquiátrica, el cuerpo humano es considerado como un acumulador de experiencias de la vida de la persona. Esto indica que tanto las experiencias de los primeros estadios de la vida, así como las frustraciones actuales pueden dejar marcas en el organismo y en las funciones corporales. Por ello, cualquier alteración mental puede repercutir en el aspecto físico del individuo (Alexander, 1987; McDougall, 1985) La profesora de fisioterapia de la Universidad de Umeå, la Dra. Mattsson (Mattsson, 1998) defiende un concepto del cuerpo que expresa la propia historia de la vida, es decir, las tensiones y los conflictos son expresados tanto en la función psicomotora, como en la postura, la marcha y la respiración. La profesora de fisioterapia psiquiátrica de la Universidad de Lund (Suecia), la Dra. Amanda Lundvik Gyllensten (Lundvik, 2001) afirma que una mayor conciencia y control del cuerpo pueden incrementar la habilidad de la persona para tolerar y expresar sentimientos y emociones. La realización de movimientos y ejercicios físicos centrados en la activación de los recursos sanos de la persona inducen a la mejora tanto la capacidad de interacción como también las funciones tanto físicas como psíquicas. Esta afirmación ha sido planteada en diversos estudios (Mattsson, 1995).

La fisioterapia psiquiátrica se basa en 4 elementos principales (Mattsson, 1994):

- Regulación de la tensión muscular mediante relajación.

- Terapia de la conciencia corporal.

- Terapia psicomotora.

- Fisioterapia activadora.

- Terapia expresiva.

Terapia de la Conciencia Corporal (TCC): La TCC es el nombre común de diversos enfoques fisioterapéuticos orientados al cuerpo y que usan una perspectiva holística durante el proceso terapéutico (Roxendal, 1985). El término TCC fue usado por primera vez a finales de la década de 1960 en relación a la fisioterapia a pacientes con trastornos neuróticos (Roxendal, 1985). En la práctica, la TCC difiere de la fisioterapia tradicional debido a que la primera presta un gran interés en la 
estimulación de la conciencia sensorial y por mostrar la máxima atención en cómo se realizan los movimientos y no en el número de repeticiones de un movimiento.

A principios de la década de los 70, Roxendal conoció al psicoanalista francés y profesor del movimiento Jacques Dropsy (2001). Descubrió el gran valor de la práctica del movimiento de Dropsy quien desarrolló el concepto de "psicotonía" basado en movimientos basales. Roxendal introdujo este concepto en la práctica fisioterapéutica bajo el nombre de Terapia de la Conciencia Corporal Basal (TCCB). Así fue como Roxendal desarrolló dos ramas de la Conciencia Corporal, una fue la TCC expresiva, en la cual el paciente trabajaba con su estilo personal especial del movimiento, comportamiento e interacción y, la otra, la TCCB, en la cual el paciente trabajaba las funciones básicas del movimiento en relación a la postura, coordinación, respiración libre y conciencia (Lundvik, 2001).

La TCC se basa en la teoría de las cuatro dimensiones existenciales, las cuales, de acuerdo con Dropsy, representan una característica esencial del ser humano. Dos dimensiones representan a los aspectos físicos, mientras que las otras dos se refieren a aspectos mentales. Éstas son:

1. Dimensión física (o estructural/material), representa al cuerpo físico incluyendo huesos, músculos, nervios, vasos sanguíneos, etc.

2. Dimensión fisiológica, representa los procesos fisiológicos vitales como la respiración, la circulación sanguínea, el sistema nervioso central, el metabolismo, etc.

3. Dimensión psicológica (o relacional), representa los pensamientos y sentimientos tanto propios como en interacción con otras personas y la vida socio-cultural.

4. Dimensión existencial, representa la habilidad de autoconciencia y autorazonamiento, según Dropsy se trata de una dimensión espiritual. Es la dimensión puramente humana, la característica que diferencia al ser humano del resto de los animales.

Dropsy plantea que cuando las cuatro dimensiones de la persona están en armonía, se puede observar una función psicotónica completa. Esta condición requiere equilibrio, un estado psicológico equilibrado y auto-presencia, formando un estado ideal que se puede experimentar con mucha satisfacción por parte de la persona, pero puede llegar a desaparecer con bastante facilidad. Mediante la práctica de la TCC estos momentos de bienestar se pueden llegar a disfrutar con mucha mayor duración. Finalmente, se debe intentar conseguir que esta experiencia se observe también durante las actividades de la vida diaria (Skatteboe, 2005)

Según Dropsy (1998) y avalado por varios fisioterapeutas (Mattsson, 1995; Skjaerven, 2003; Skjaerven, 2002a) existen tres factores principales para la integración de las cuatro dimensiones del movimiento. Su función es actuar como puentes uniendo las diferentes dimensiones. Éstos son equilibrio, respiración libre y conciencia, tres componentes que constituyen la esencia de la TCC (ver tabla 1).

Tabla 1.

Componentes del movimiento (Skjarven, 2003)

\begin{tabular}{ccc}
\hline Equilibrio & Respiración libre & Conciencia \\
\hline Estabilidad postural & Flujo & Centrado \\
\hline Coordinación & Ritmo del movimiento & Unidad del movimiento \\
\hline Espacio & Tiempo & Energía \\
\hline
\end{tabular}


En 1985, Roxendal, publicó su Tesis Doctoral en el Departamento de Rehabilitación Médica de la Universidad de Gotemburgo (Suecia) y fue titulada: "Body Awareness Therapy and the Body Awareness Scale. Treatment and Evaluation in Psychiatric Physiotherapy" (Terapia de la Conciencia Corporal y la Body Awareness Scale. Tratamiento y evaluación en fisioterapia psiquiátrica). Esta fue la primera Tesis Doctoral publicada en el campo de la Fisioterapia en Psiquiatría y Salud Mental. Roxendal quiso demostrar que la fisioterapia psiquiátrica y la conciencia corporal pueden aplicarse en clínicas o departamentos de Psiquiatría y Psicosomática. También desarrolló un método de evaluación dirigido a pacientes psiquiátricos. Describió la TCC y abordó el marco conceptual de términos corporales tales como "conciencia corporal", "experiencia corporal", "patrón del movimiento y disfunción corporal general", etc. También desarrolló la Body Awareness Scale (BAS) (Escala de Conciencia Corporal), siendo una combinación de ítems de la "Comprehensive Psychopathological Rasting Scale", unida a nuevos ítems corporales.

En la década de 1990, se creó el grupo de profesores de la TCC, el cual está organizado para promover esta disciplina entre los fisioterapeutas a nivel internacional (ver www.ibk.nu). Desde entonces, han estado desarrollando las teorías, práctica e investigación de la TCC. Hoy día la TCC se encuentra introducida en los planes de pregrado de Fisioterapia en las universidades de los países nórdicos y forma parte de las competencias profesionales de los fisioterapeutas (Mattsson, 1998).

En 1998, la fisioterapeuta y profesora Dra. Monica Mattsson publicó su tesis doctoral (Mattsson, 1998). titulada "Body Awareness. Applications in physiotherapy" (Conciencia Corporal. Aplicaciones en Fisioterapia). Fue realizada en el Departamento de Psiquiatría y Medicina de Familia de la Universidad de Umeå (Suecia). El punto de partida de dicha tesis fue la actitud hacia el cuerpo en los campos de Psiquiatría y Psicosomática. El objetivo fue ofrecer una descripción y evaluar aplicaciones de la fisioterapia psiquiátrica dentro de la psiquiatría y psicosomática. La conciencia corporal es tratada como el concepto central de esta Tesis Doctoral. Por ello concluyó afirmando que los fisioterapeutas especialistas en salud mental pueden ofrecer mediante la TCC una contribución adicional en el diagnóstico psiquiátrico y psicológico, y ofrecer mayores alternativas en el tratamiento (Mattsson, 1998). La tesis demuestra que la fisioterapia psiquiátrica a través del trabajo de la conciencia corporal es de gran utilidad en los campos de Psiquiatría y Psicosomática. Concluye demostrando que este enfoque de la fisioterapia puede tener una importancia creciente en el sistema de salud (Mattsson, 1998).

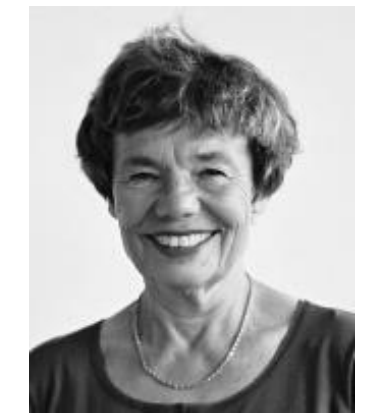

Figura 5. Monica Mattsson

La fisioterapeuta y profesora Dra. Amanda Lundvik Gyllensten publicó su Tesis Doctoral titulada "Basic Body Awareness Therapy. Assessment, treatment and interaction" (Terapia de la Conciencia Corporal Basal. Evaluación, tratamiento e interacción) (Lundvik, 2001). Fue leída en 2001 en el Departamento de Fisioterapia de la Universidad de Lund (Suecia). Lundvik Gyllensten enfocó su tesis 
en investigar la metodología de la Terapia de la Conciencia Corporal Basal, validando el instrumento de evaluación del movimiento "Body Awareness Scale-Health (BAS-H)" (Body Awareness Scale Health") y el comportamiento del movimiento, investigando el beneficio de la intervención del tratamiento y explorando los aspectos que interaccionan en el encuentro entre el paciente y el terapeuta. Los campos de la intervención fueron psiquiatría, rehabilitación multidisciplinar del dolor y atención primaria de salud. Los pacientes que recibieron la intervención fisioterapéutica perteneciente a psiquiatría mostraron grandes mejoras en relación al equilibrio y la postura (Lundvik, 2001).

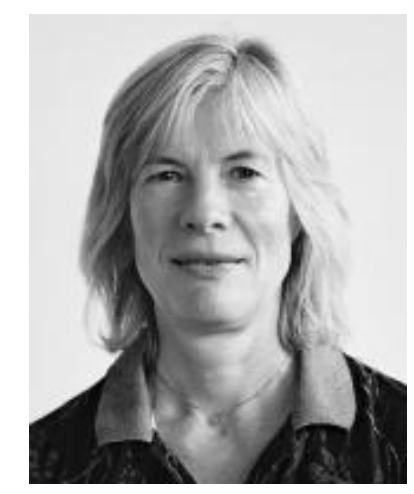

Figura 6. Amanda Lundvik-G

En el 2003, la fisioterapeuta de la Universidad de Bergen (Noruega) Liv Helvik Skjærven consiguió desarrollar un programa de posgrado universitario para fisioterapeutas en TCCB. Con una carga lectiva de 60 ECTS (Sistema Europeo de Transferencia de Créditos) dicho programa está dirigido al uso de la TCCB en los servicios de salud mediante un enfoque práctico (ver http://student.hib.no/fagplaner/ahs/basic_bam/basic_bam.pdf). Skjærven ha organizado toda la docencia en inglés con la intención de extender la TCCB fuera de las fronteras nórdicas. Por ello, fisioterapeutas de cualquier parte del mundo están actualmente formándose en esta metodología de trabajo. Al mismo tiempo ha elaborado diversas publicaciones (Skjaerven, 2003; Skjaerven, 2002a; Skjaerven, 2002b; Skjaerven, 2004) en las que presenta perspectivas teóricas y prácticas de la TCCB tanto a nivel individual como grupal y de los instrumentos de evaluación (Body Awareness Rating Scale, BARS). Skjærven ha centrado sus estudios fundamentalmente en la calidad del movimiento y en la descripción del movimiento armónico y su relación con procesos psicopatológicos. Su larga experiencia como fisioterapeuta en Psiquiatría se puede observar en las excelentes descripciones metodológicas que ofrece de la intervención fisioterapéutica mediante el uso de la TCCB y en cómo describe los aspectos cualitativos del movimiento. Hoy día es considerada como uno de los referentes internacionales de la Fisioterapia en Salud Mental y fue la Presidenta de la siguiente Conferencia Internacional sobre Fisioterapia en Psiquiatría y Salud Mental que se celebró en Bergen (Noruega) en febrero de 2008. 


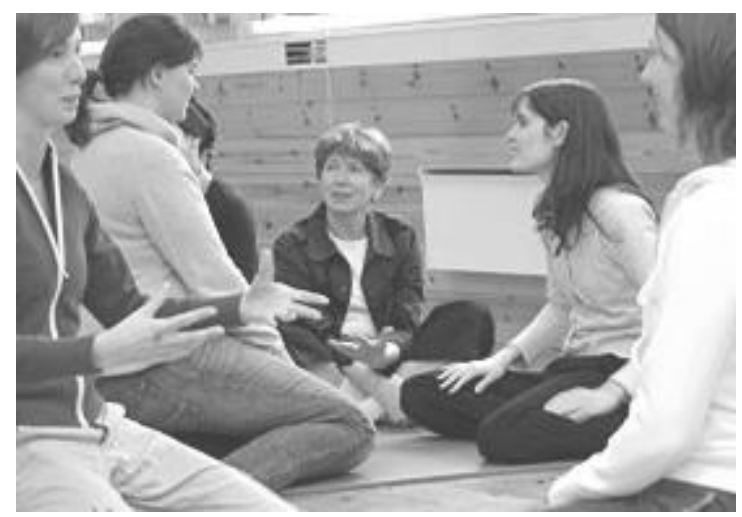

Figura 7. Liv Helvik Skjærven, en el centro.

En el 1990, la fisioterapeuta noruega Ulla-Britt Skatteboe, profesora en estudios de postgrado en Fisioterapia Psicosomática y Psiquiátrica del Departamento de Ciencias de la Salud de la Universidad de Oslo, publicó su tesis del Master en Fisioterapia titulada "Fisioterapia grupal para pacientes con neurosis crónica y desórdenes de la personalidad" (Friis et al, 1989; Skatteboe, Friis, Kvamsdal y Vaglum, 1989) En 2005, publicó su proyecto titulado "Basic Body Awareness Therapy and Movement Harmony" (Terapia de la Conciencia Corporal Basal y Armonía del movimiento). En él presenta sus experiencias trabajando con la TCC durante diez años. Al mismo tiempo describe un extenso marco conceptual de la teoría del movimiento humano, y también desarrolla en colaboración con Skjærven y otros fisioterapeutas, la "Body Awareness Scale" (Body Awareness Rating Scale). Realiza un profundo análisis sobre los puntos de observación del movimiento humano y presenta los 12 movimientos basales en los que las cuatro dimensiones del movimiento humano están representadas.

Otras corrientes en Fisioterapia en Salud Mental: Michel Probst, fisioterapeuta nacido en Köln (Alemania) pero trabajando actualmente en la Universidad Católica de Lovaina (Bélgica), publicó su tesis doctoral en 1997. Fue titulada "Body experience in eating disorder patients" (Experiencia corporal en pacientes con trastornos alimentarios) y realizada en la Facultad de Educación Física y Fisioterapia de la Universidad Católica de Lovaina (Bélgica) (Probst, 1997). Ha realizado numerosos estudios sobre diversas intervenciones fisioterapéuticas en pacientes que sufren trastornos alimentarios. Se trata de una referencia básica para la segunda parte de esta tesis. Probst desarrolló el famoso y reconocido internacionalmente "Body Attitude Test" (Test de la actitud corporal) muy utilizado actualmente en pacientes con trastornos alimentarios. Elaboró el método de vídeo-distorsión y un amplio estudio entre la evaluación de la composición corporal, la relación entre la experiencia corporal y otras variables y diversas líneas de tratamientos basadas en experiencias corporales y dirigidos a pacientes con trastornos alimentarios (Probst, 1997). Fundamentalmente ha usado la fisioterapia psicomotora como técnica de tratamiento unida a otros enfoques adaptados a cada tipo de paciente, como el método antes mencionado de video-distorsión.

La fisioterapeuta británica Mary Hare publicó en 1986 su libro titulado "Physiotherapy in Psychiatry" (Fisioterapia en Psiquiatría) (Hare, 1986). En la obra ofreció una visión global de la fisioterapia en el campo de la salud mental, junto a unos fundamentos básicos en psiquiatría clínica. Presentó multitud de casos en los que se ha aplicado fisioterapia a pacientes de departamentos de salud mental y las dificultades observadas en el tratamiento a este tipo de pacientes. En este texto se analizan técnicas tales como relajación, ejercicio y auto-ayuda, reflejando un aumento de conciencia de la relación entre la mente y el cuerpo. 
Entre 1995 y 2003, la fisioterapeuta británica Tina Everett, supervisora del servicio de fisioterapia del servicio de salud mental de Oxford (Inglaterra), en colaboración con diversos expertos en Psiquiatría y Salud Mental, edita dos libros titulados respectivamente "Physiotherapy in Mental Health" (Fisioterapia en Salud Mental) y "Interventions for Mental Health. An Evidence-based Approach for Physiotherapists and Ocupational Therapists" (Intervenciones en Salud Mental. Un enfoque basado en la evidencia para fisioterapeutas y de terapia ocupacional) (Everett et al, 2003; Everett et al, 1995). En el libro se abordan diversas estrategias fisioterapéuticas para alteraciones mentales y se expone el grado de evidencia científica que se dispone en las mismas, tratando de diversos temas en relación a la actuación fisioterapéutica, tales como demencia, adiciones, trastornos alimentarios, síndrome de fatiga crónica, abuso sexual infantil, autolesiones, estrés, dolor crónico, entre otros.

Tal es el creciente interés mostrado por los fisioterapeutas en Psiquiatría y Salud Mental que en 2006 se celebró la I Conferencia Internacional sobre Fisioterapia en Psiquiatría y Salud Mental. Se realizó en Lovaina (Bélgica) siendo el fisioterapeuta Michel Probst el organizador de las mismas. A ella acudieron 122 fisioterapeutas de 21 nacionalidades diferentes y se presentó una amplia gama de intervenciones fisioterapéuticas realizadas en el campo de la salud mental.

\section{Reflexiones finales}

Los antecedentes históricos de la Fisioterapia en Salud Mental hacen que esta especialidad cuente con un ente de conocimiento propio, ofreciendo al fisioterapeuta un amplio campo teórico-conceptual. Este grupo de conocimientos y experiencias previas constituyen el punto de partida para todo aquel fisioterapeuta que desee comenzar a desarrollar experiencias prácticas y/o ensayos clínicos sobre esta especialidad en auge: Fisioterapia en Salud Mental.

\section{Conflicto de Intereses}

El autor declara no presentar ninguna relación de interés comercial o personal dentro del marco de la investigación que condujo a la producción del manuscrito.

\section{Referencias}

Alexander, F. (1932) The use of the self. Los Angeles, Estados Unidos: Centerline Press.

Alexander, F. (1987).The use of the self. Nueva York, Estados Unidos: Dutton.

Baril, J. (1987). La danza moderna. Barcelona, España: Paidós.

Baumann, J. (1994). Körperbezogene Gruppenpsuchotherapie in der 2. Lebenshälfte. Psychotherapie, Psychosomatic und medizinische Psychologie, 44, 337-345.

Bott, V. (2006) Medicina Antroposófica. Una ampliación del arte de curar. Madrid: Luis Carcomo Ed.

Bunkan, B. H. (1979). Undersökelse og behandling ved muskelspenninger. Oslo, Noruega: Universitetsforlaget.

Degener A. (2006). The body-concept of adolescents with self-injurious behaviour. Libro de resúmenes de la I Conferencia Internacional de Fisioterapia en Psiquiatría y Salud Mental. Universidad Católica de Lovaina, Bélgica.

Deter, H. C \& Allert, G. (1983). Group therapy for Asthma patients: a concept for the psychosomatic treatment of patients in a medical clinic - a controlled study. Psychotherapy and Psychosomatics, 40, 95-100. https://doi.org/10.1159/000287757 
Donaghy M, Durward B. (2000). A report on the clinical effectiveness of physiotherapy in mental health. Londres, Inglaterra: Chartered Society of Physiotherapy.

Dragesund, T. \& Raheim, M. (2006). Body consciousness and Norwegian psychomotor physiotherapy. Experiences from patients with chronic widespread pain. Libro de resúmenes de la I Conferencia Internacional de Fisioterapia en Psiquiatría y Salud Mental. Universidad Católica de Lovaina. Bélgica. https://doi.org/10.1037/e573062012-040

Dropsy J. (1998). Human expression, the coordination of mind and body. En L.H. Skjaerven. Quality of Movement - the Art and Health. Lectures on Philosophy, Theory and Practical Implications to Basic Body Awareness Therapy. Bergen, Noruega. p. 8-20.

Dropsy J. (2001). Human expression the coordination of mind and body. En Lundvik Gyllensten, A. (2001). Basic Body Awareness Therapy. Assessment, treatment and interaction. Tesis doctoral, Universidad de Lund. Suecia. p. 17

Duncan I. (1938). Mi vida. Buenos. Aires, Argentina: Losada.

Everett, T., Dennis, M. \& Rickets, E. (1995). Physiotherapy in Mental Health. Oxford, Inglaterra: Butterworth Heinemann.

Everett, T., Donaghy, M. \& Feaver, S. (2003). Interventions for Mental Health. An Evidence-based Approach for Physiotherapists and Occupational Therapists. Oxford, Inglaterra: Butterworth Heinemann.

Fary, R. (2003) Increased work value. The case for Physiotherapy 2004. Sydney, Australia: Australian Association of Physiotherapy

Fisher, S. \& Cleveland, S. E. (1958) Body image and personality. Nueva Jersey, Estados Unidos: S van Noostrand Co Inc.

Freud, S. (1923). El Yo y el Ello. Madrid, España: Amorrortu Editores XIX.

Friis, S., Skatteboe, U. B., Kvamsdal Hope, M. \& Vaglum, P. (1989). Body awareness group therapy for patients with personality disorders - evaluation of the body awareness rating scale. Psychotherapy and Psychosomatics, 51, 18-24. https://doi.org/10.1159/000288129

Goertzel, V., May, P.R., Salkin, J. \& Schoop, T. (1965). Body-ego technique: an approach to the schizophrenic patient. Journal of Nervous and Mental Disease, 141, 53-60.

Gudat, U., Convent, G. \& Heinemann, L. (1997). Metformin and exercise: no additive effect on blood lactate levels in health volunteers. Diabetic Medicine, 14, 138-42. https://doi.org/10.1002/(sici)1096-9136(199702)14:2<138::aid-dia311>3.0.co;2-s

Hare, M. (1986). Physiotherapy in Psychiatry. Londres, Inglaterra: Heinemann Physiotherapy.

Hulting, K. (2006). Negative body image among young women with borderline personality disorder. Libro de resúmenes de la I Conferencia Internacional de Fisioterapia en Psiquiatría y Salud Mental. Universidad Católica de Lovaina, Bélgica. https://doi.org/10.1037/e573062012-036

Johnsen, L. (1969). Integrert respirationsterapi. Oslo, Noruega: Universitetsforlaget.

Laban, R. (1960). The mastery of movement. Boston, Estados Unidos: Macdonald \& Evans.

Larisch, A. (2006). Tanztherapie aus der Sicht von Patienten. En: Loew TH, Tritt K, Lahmann C, Röhricht F. Body psychotherapy - scientifically proved? An overview of empirically evaluated body oriented psychological therapies (en alemán). Psychodynamische Psychotherapie; 5:6-19.

Loew, F. \& Rapin, C.H. (1994). The paradoxes of quality of life and its phenomenological approach. Journal of Palliative Care, 10, 37-41. https://doi.org/10.1177/082585979401000109

Loew, T.H. \& Siegfried, W., Martus, P., Tritt, K. \& Hahn, E. G. (1996). Functional relaxation reduces acute airway obstruction in asthmatics as effectively as inhaled terbutaline. Psychotherapy and Psychosomatics, 65, 124-128. https://doi.org/10.1159/000289063

Loew, T.H., Sohn, R., Martus, P., Tritt, K. \& Rechlin, T. (2000). Functional relaxation as a somatopsychotherapeutic intervention: a prospective controlled study. Alternative Therapies, Health and Medicine, 6, 70-75. 
Loew, T.H., Tritt, K., Siegfried, W., Bohmann, H., Martus, P. \& Hahn, E.G. (2001). Efficiacy of "functional relaxation" method in patients with acute asthma. A randomised, prospective, controlled, crossover experimental investigation. Psychotherapy and Psychosomatics, 70, 151157. https://doi.org/10.1159/000056241

Lowe, T.H., Tritt, K., Lahmann, C. \& Röhricht, F. (2006). Body psychotherapy - scientifically proved? An overview of empirically evaluated body oriented psychological therapies (en alemán). Psychodynamische Psychotherapie, 5, 6-19.

Lowen, A. (1975) Bioenergetics. The revolutionary therapy that uses the language of the body to heal the problems of the mind. Coward McCann \& Geoghean Inc.

Lowen, A. (1988). Ejercicios de bioenergética. Málaga: Sirio.

Lowen, A. (1995). El lenguaje del cuerpo. Barcelona, España: Herder.

Lundvik Gyllensten, A. (2001) Basic Body Awareness Therapy. Assessment, treatment and interaction. Tesis doctoral. Universidad de Lund. Suecia.

Mattsson, M., Egberg, K., Armelius, K. \& Mattsson, B. (1995). Long-term of physiotherapeutic treatment in outpatient psychiatric care. Nordic Journal of Psychiatry, 49,103-110. https://doi.org/10.3109/08039489509011891

Mattsson, M. \& Mattsson, B. (1994). Physiotherapeutic treatment in out-patient psychiatric care. Scandinavian Journal of Caring Sciences, $\quad 8, \quad 119-126 . \quad$ https://doi.org/10.1111/j.14716712.1994.tb00241.x

Mattsson, M. (1998). Body Awareness and applications in physiotherapy. Tesis doctoral. Umeå. Universidad de Umeå. Suecia

Maurer-Groeli YA. (1976). Körperzentrierte Gruppenpsychotherapie bei akut schizophren Erkrankten. Eine Untersuchung mittels Ich-Funktionen Rating nach Bellak. (artículo en alemán). Archiv der Psychiatrie und Nervenkrankheiten, 221, 259-71. https://doi.org/10.1007/bf00418484

McDougall J. (1985). Theatres of the mind. Illusion and truth on the psychosomatic stage. New York, Estados Unidos: Basic Books

Merleau-Ponty M. (1962). Phenomenology of perception. Londres, Inglaterra: Routledge.

Nitsun, M., Stapleton, J. H. \& Bender, M. P. (1974). Movement and drama therapy with long-stay schizophrenics. British Journal of Medical Psychology, 47, 101-19. https://doi.org/10.1111/j.2044-8341.1974.tb02275.x

Probst, M. (1997). Body experience in eating disorder patients. Tesis doctoral. Lovaina, Katholieke Universiteit Leuven. Bélgica

Reich, W. (1970). Reich habla de Freud. Barcelona, España: Ed. Anagrama.

Reich, W. R. (1949). Character Analysis. $3^{a}$ ed. Nueva York, Estados Unidos: Simon and Schuster.

Roxendal, G. (1985) Body Awareness Therapy and the Body Awareness Scale, treatment and evaluation in psychiatric physiotherapy. Tesis doctoral. Universidad de Gotemburgo. Suecia

Schilder, P. (1950) The image and appearance of the human body. Nueva York, Estados Unidos: Intern Univ Press.

Skatteboe, U. B, Friis, S., Kvamsdal Hope, M. \& Vaglum, P. (1989). Body awareness group therapy for patients with personality disorders. Description of the therapeutic method. Psychotherapy and Psychosomatics, 51, 11-17. https://doi.org/10.1159/000288128

Skatteboe, U. B. (2005). Basic Body Awareness Therapy and Movement Harmony. Oslo, Noruega: Oslo University College.

Skjaerven, L.H. (1999). A vare seg selv - helt fullt og helt. En feltstudie av Bevegelseskvalitet. Bergen, Noruega: Hovedfagsoppgave UiB.

Skjaerven, L. H. (2002a). Basic Body Awareness Therapy. Exercises, verbal guidance, observation and assessment of Quality of Movement. A first introduction. Bergen Noruega.

Skjaerven LH. (2002b). Quality of movement - the art and health. Bergen Noruega. 
Skjaerven, L.H. (2003). Basic Body Awareness Therapy. A guide to understanding, therapy and growth. Bergen. Noruega

Skjaerven, L.H. (2004). Being in dialogue. Basic body Awareness in group therapy. Bergen Noruega. Skjaerven, L.H. (2006). Physiotherapy in Mental Health - A Scandinavian approach. Libro de resúmenes de la I Conferencia Internacional de Fisioterapia en Psiquiatría y Salud Mental. Universidad Católica de Lovaina, Bélgica

Stanislavski, K. (1962). My life in art. New York, Estados Unidos: Theater Arts Books.

Steiner, R. (1974). Eurythmy and the impulse to dance. Donarch, Suiza: Rudolf Steiner Press.

Ventling, C. D \& Gerhard, U. (2000) Zur Wirksamkeit bioenergetischen Psychotherapien und Stabilität des Therapieresultats: Eine retrospektive Untersuchung. Psychotherapeut, 45, 230-236

Yusta, M. (1996) Aportación a una definición de Fisioterapia. En: X Congreso Nacional de Fisioterapia. Salamanca: Viajes y Congresos. p. 106

\section{Información de autor:}

Daniel Jesús Catalán Matamoros

Fisioterapeuta, $\mathrm{PhD}$ en Fisioterapia

Universidad Carlos III de Madrid

Universidad de Almería.

dcatalan@ual.es

https://orcid.org/0000-0002-3086-6812 